\title{
Fiber-Based Interferometry and Imaging
}

\author{
E. Serabyn*a, K. Liewer ${ }^{\mathrm{a}}$, S.R. Martin ${ }^{\mathrm{a}}$, D. Mawet ${ }^{\mathrm{b}}$, and A. Ksendzov ${ }^{\mathrm{a}}$ \\ a Jet Propulsion Laboratory, California Institute of Technology, Pasadena, CA, USA 91109; \\ ${ }^{b}$ European Southern Observatory, Alonso de C'ordova 3107, Vitacura 7630355, Santiago, Chile
}

\begin{abstract}
Single-mode optical fibers are playing an increasing role in astronomical interferometry, e.g., in high-accuracy visibility measurements and in nulling interferometry. However, such observing modes typically involve only small numbers of fibers. On the other hand, some recently proposed observing techniques call for arrays of single mode fibers coupled to arrays of sub-apertures within a large telescope pupil. The concepts include pupil-masked visibility measurements (nonredundant masking), pupil-sheared nulling interferometry, and coronagraphic imaging using a fiber-linked phased-array of small optical telescopes. The latter arrangement may also be relevant to optical communications. Here we provide an overview of a number of recent novel applications of single-mode fibers and single-mode fiber arrays.
\end{abstract}

Keywords: optical fibers, nulling interferometry, coronagraphy, pupil remapping

\section{INTRODUCTION}

The imaging and spectral characterization of arbitrary planets orbiting nearby stars remains extremely difficult because of small star-planet separations and miniscule planet/star contrast ratios. Indeed, the goal of imaging terrestrial exoplanets is very likely beyond the reach of the current generation of ground-based and space-based telescopes. In particular, state-of-the-art telescopes have only recently enabled the imaging of four unusually bright jovian planets ${ }^{1}$ around the star HR8799 at contrasts relative to their host star of a few $10^{-5}$. Next generation adaptive optics systems on large ground based-telescopes are expected to soon take the next step to the range of $\sim 10^{-6}$ to $10^{-8}$ contrast, with the exact level to be attained dependent on various atmospheric and instrumental factors. Reaching the $10^{-10}$ contrasts needed for terrestrial exoplanet detection will thus almost certainly require observations from space, in order to avoid the effects of the fluctuating atmosphere.

To enable observations at small angular separations from stars, large telescopes are required, but large, highly-accurate space telescopes are very expensive, with costs scaling roughly as the diameter to the $2.5^{\text {th }}$ power ${ }^{2}$. The development of novel, low-cost telescope architectures able to provide a high degree of stellar rejection and to reach high contrast levels would thus be of great interest.

In response to these technical and financial challenges, a number of novel interferometric and coronagraphic approaches to starlight suppression and small-angle observations have been proposed recently. Several of these approaches rely on the unique properties of single-mode optical fibers, including their ability to transport light to arbitrary locations, their spatial filtering capability ${ }^{3}$ (Fig. 1), their ability to control transmitted intensities by means of fiber-coupling (alignment) modifications, their ability to be used as beam combiners and splitters, and their ability to reconfigure, or remap, the distribution of light in a pupil plane. Progress toward demonstrating some of these novel fiber-based techniques in the laboratory and on the sky is being made in several areas. Here we briefly summarize recent progress and new concepts in fiber-based approaches in astronomical interferometry and high-contrast imaging.

\section{USES OF FIBERS IN ASTRONOMICAL INTERFEROMETRY}

In two-beam interferometry, single-mode fibers can be used to yield a higher level of visibility calibration accuracy by spatially filtering either the combined (Fig. 2, top) or the incoming (Fig. 2, center) wavefronts. The former is made use of in systems such as the Keck Interferometer fringe tracker ${ }^{4}$, where the fiber acts only as a spatial filter, and the latter is used by, e.g., the FLUOR beam-combiner ${ }^{5}$. In the latter case, the beam-combination is also fiber-based (Fig. 2, center), and additional fiber branchings also allow for individual beam intensity measurements. These approaches extend

UV/Optical/IR Space Telescopes and Instruments: Innovative Technologies and Concepts V, edited by Howard A. MacEwen, James B. Breckinridge, Proc. of SPIE Vol. 8146, 81460I - (c) 2011 SPIE CCC code: $0277-786 \mathrm{X} / 11 / \$ 18 \cdot$ doi: $10.1117 / 12.895268$ 
naturally to integrated optics, e.g., the case of PIONIER four-beam combiner ${ }^{6}$, in which fibers feed an integrated optics beamcombiner chip. The PIONIER beam-combiner also incorporates phase-shifting elements to allow all four quadrature phases to be measured simultaneously in parallel outputs on all six baselines.

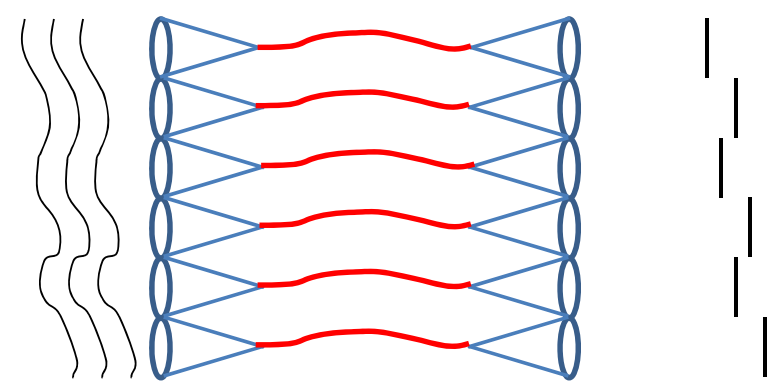

Figure 1. Illustration of the spatial filtering property of single mode fibers. Only the average phase in each subaperture propagates through the single mode fibers.

A lone fiber can also play the role of a beam-combiner (Fig. 2, bottom), if two parallel beams are injected into the same fiber with a common optic, because both beams must couple into the same fiber mode. Moreover, a single fiber can also play the role of a nulling beamcombiner ${ }^{7,8}$, if a relative $\pi$-radian phase shift is applied to the two input beams prior to injection. In this case, the two oppositely directed electric fields coupling into the fiber mode cancel each other, and so cannot propagate. However, off-axis light from the planet arriving with a different relative phase shift between the beams still propagates down the fiber to the detector. This approach is employed by the Palomar Fiber Nuller ${ }^{9}$ (PFN).

High accuracy is especially important in nulling interferometry, and it has recently been demonstrated that rather than expending effort to accurately stabilize the null depth, one can instead extract accurate null depths from fluctuating null depth data by modeling the statistics of the fluctuations ${ }^{10}$, an approach that has enabled the PFN to achieve null depth accuracies of $<0.1 \%$.
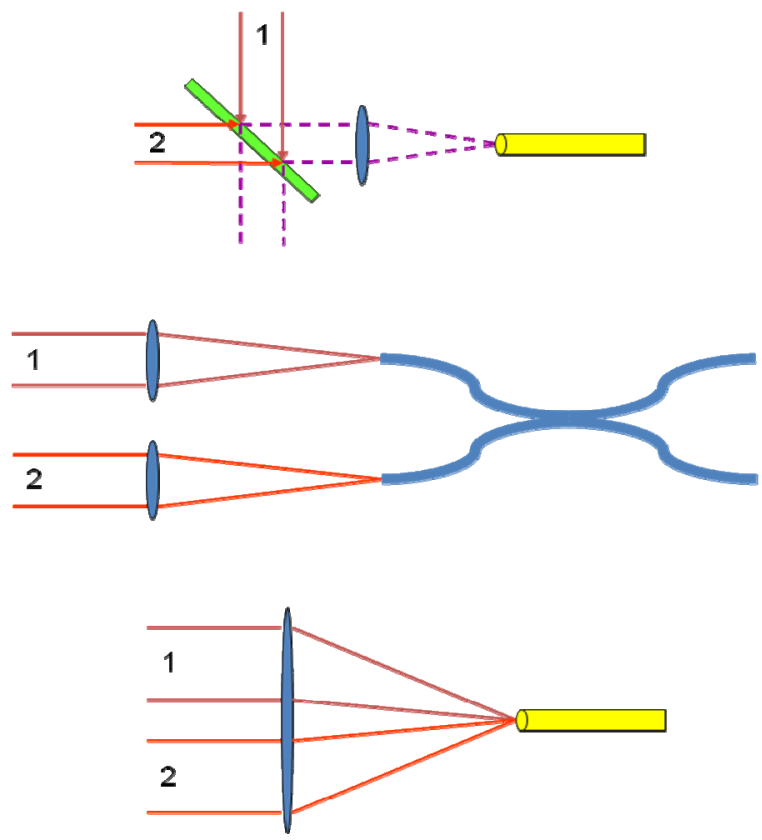

Figure 2. Configurations of fiber-based interferometric beam combiners and spatial filters. 
Finally, it should also be mentioned that the PFN makes use of a baseline that can be rotated within the Hale telescope's pupil, thus enabling a rotating nuller as originally envisioned for space-based nulling interferometry aimed at exoplanet detection ${ }^{11}$. Such techniques are also applicable to larger telescopes such as the Keck telescope, and someday to the Thirty Meter Telescope, eventually allowing observations within $\approx 4$ mas of bright $\operatorname{stars}^{12}$.

\section{FIBER ARRAYS}

There are currently three main astronomical observing modes under consideration that rely on the use of arrays of singlemode fibers. These all involve sub-aperturing a large pupil area, and include both interferometric and imaging applications. They all rely on the fiber's spatial filtering ability, which means that single-polarization, monochromatic light will retain only piston errors after passage through a fiber array (Fig. 1). For a point source at a given off-axis angle, a stair-step wavefront then emerges from the fiber array, with the phase of each step given by the average phase of that subaperture's input wavefront. The three techniques are compared at the block diagram level in Fig. 3, and are discussed in more detail in the following sections.

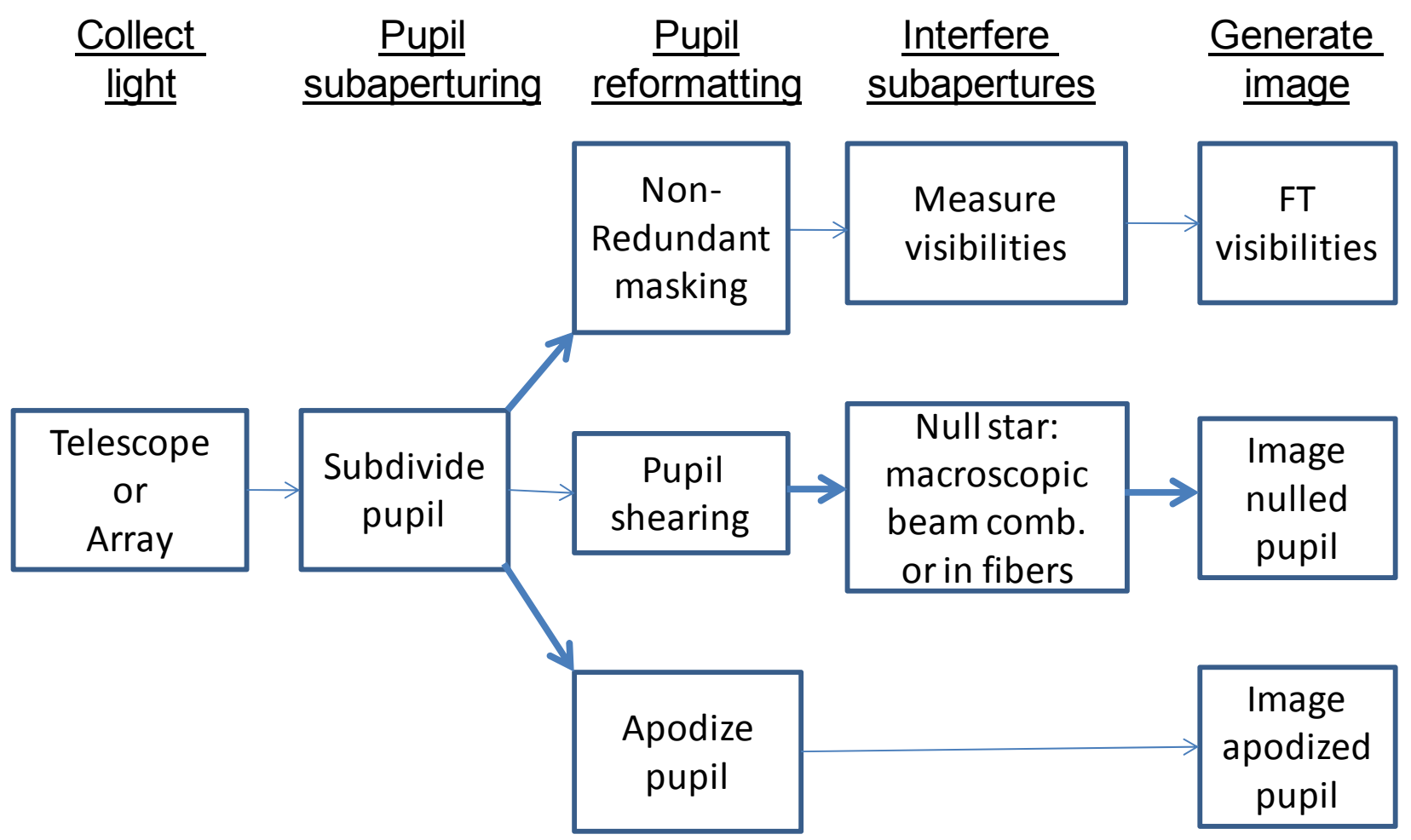

Figure 3. A block-diagram comparison of fiber-array-based imaging and interferometry approaches. The darker arrows indicate where fiber arrays play a role. In the "pupil shearing/null star" sequence, the fiber array can either follow free space nulling beam combiners, or the pupil shearing and nulling can potentially also be carried out in fibers.

\subsection{Pupil masking}

Pupil masking is a technique that simultaneously measures interferometric fringe visibilities on a large number of baselines between subapertures lying within a large telescope aperture. Non-redundant arrays of subapertures are typically used to maximize and orthogonalize the information collected. This technique has already been applied successfully on large telescopes without the use of fibers, and provides a unique observational capability ${ }^{13}$. 
The performance achievable by non-redundant pupil masking is expected to improve when each of the subapertures is spatially filtered by passage through a single-mode fiber, leading to improved closure phases and visibilities ${ }^{14}$. Moreover, the use of an array of single mode fibers would bring additional benefits, such as the ability to transform a redundant array configuration into a downstream non-redundant array configuration, in order to ease the post-detection separation of the visibility data on the different baselines, and the ability to remap a 2-dimensional pupil into a linear non-redundant array for spectroscopic purposes. Fiber-based pupil remapping thus allows one to combine the advantages of large numbers of baselines (and a large total collecting area) with a high visibility measurement accuracy, thus allowing higher contrast levels to be reached. The FIRST project ${ }^{15}$ is developing these approaches, with plans to deploy systems with up to 36 subapertures. Laboratory testing has already demonstrated the ability to recover an off-axis source from the visibility data collected in this approach ${ }^{16}$.

\subsection{Shearing Nulling Coronagraphy}

Lateral-shearing nulling interferometry has been proposed as a means of providing the high degree of stellar rejection needed to detect terrestrial exoplanets at visible wavelengths ${ }^{17}$. The idea is to superpose a set of four $\mathrm{x}$ - and $\mathrm{y}$-shifted pupils, null the starlight in the pupil overlap region, and then make a final image with a common optic that focuses the residual light in the pupil overlap region. To achieve deep stellar rejection in each subaperture in the presence of input wavefront errors, a deformable mirror with elements matched to the elements of the fiber array is used to individually phase each subaperture.

Prototype fiber assemblies have been manufactured for use in demonstrating this technique ${ }^{18}$, and progress is being made toward system-level demonstrations on laboratory testbeds, such as the APEP ${ }^{19}$ testbed at JPL and the VNT ${ }^{20}$ testbed at Goddard. Fig. 4 shows the intensity and phase outputs of a recent fiber array measured at JPL (Sandhu, priv. comm.).
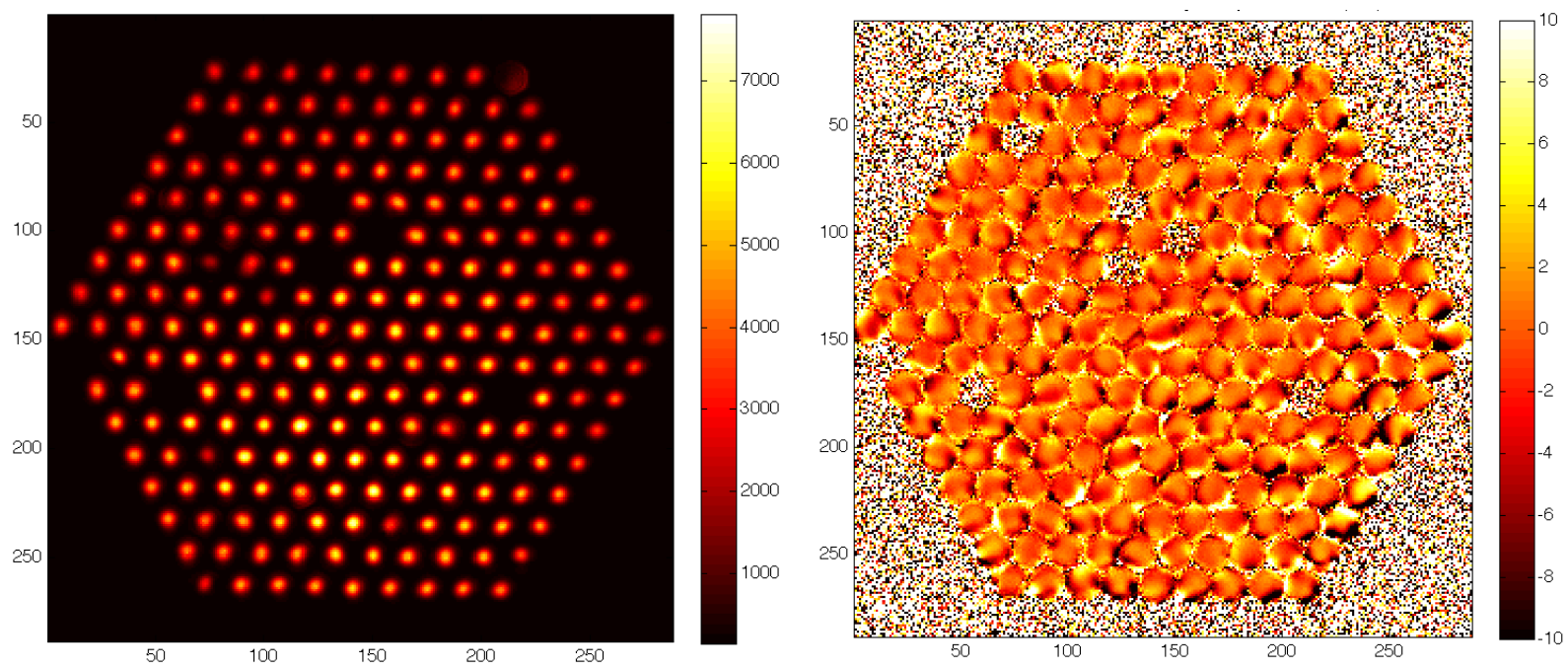

Figure 4. A 217 element prototype fiber array (Sandhu, priv. comm.) manufactured for the APEP testbed by Fiberguide Industries. The left panel shows the intensity distribution, while the right panel shows the residual phase errors (in $\mathrm{nm}$ ) between two nulled beams.

\subsection{Imaging with a fiber-linked subaperture array}

Finally, another novel high-contrast imaging approach aims at reducing potential mission cost by doing away with the large and expensive monolithic space telescope in favor of an array of small, inexpensive collector telescopes ${ }^{21}$. This approach also eliminates the distant secondary mirror, leading to a compact, nearly planar configuration (Fig. 5). In principle, such an array could be folded for launch, potentially providing a large collecting area with low mass, volume and cost. 


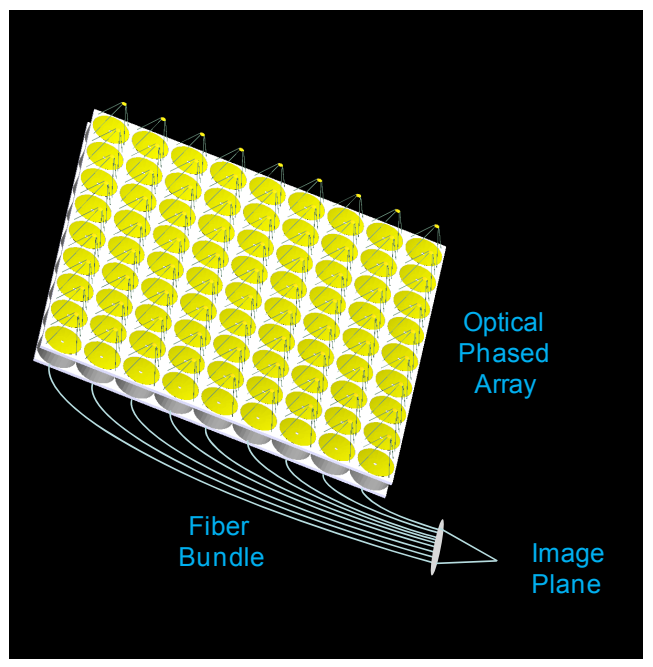

Figure 5. A close-packed fiber-linked array of small telescope apertures for small field-of-view observations.

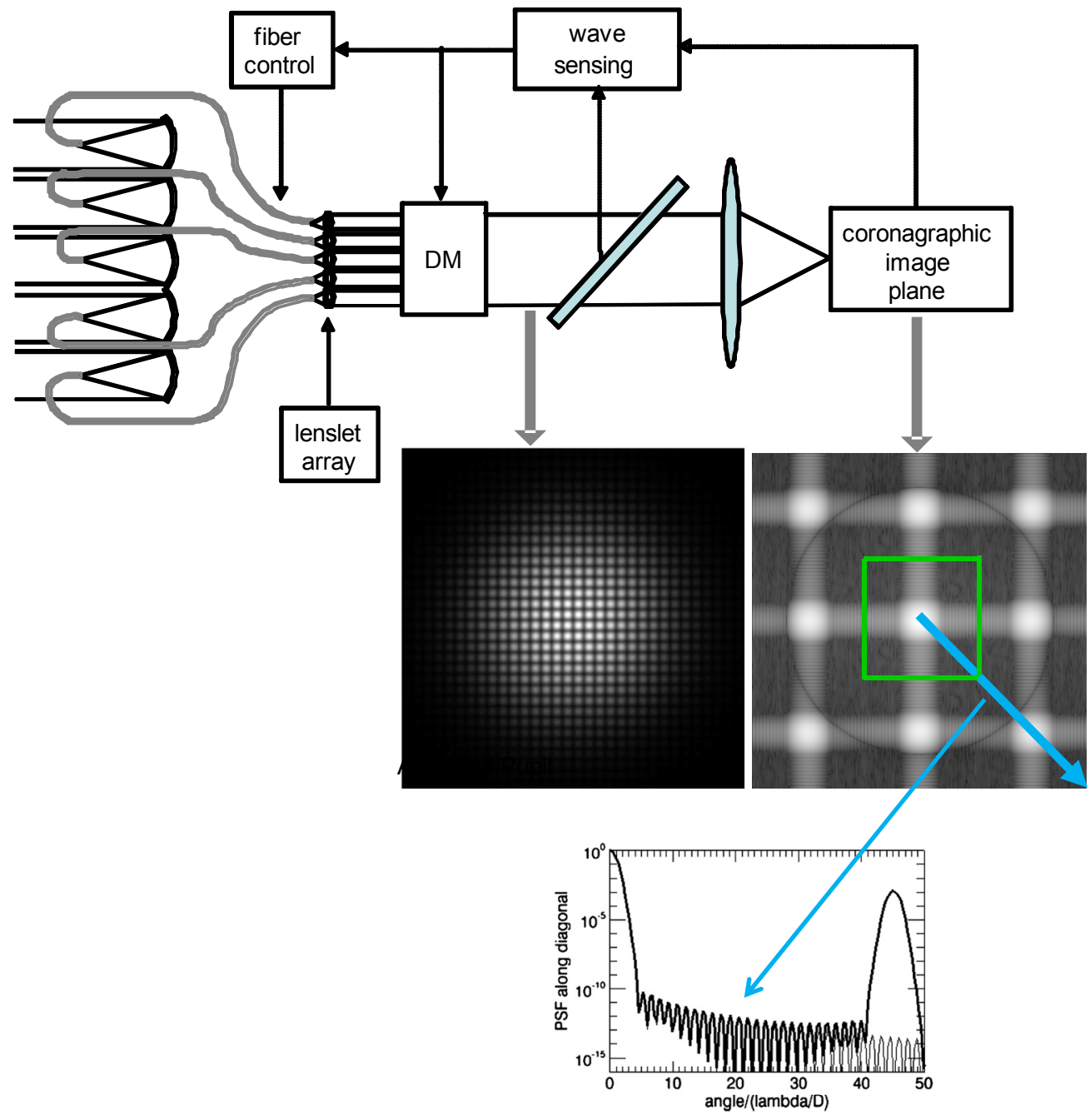

Figure 6. Phased Array Coronagraph (PAC) mission architecture and operation. 
After collection, the array of light beams can be assembled and phased into a single compressed "envelope" pupil by an array of single mode fibers coupled to a lenslet array. The assembled pupil can then be focused to a final image by a common optic (Fig. 6). Such a fiber-based relay can potentially provide complete control over the assembled pupil, i.e., over the amplitude, phase and dispersion of the light in each pupil element: phase control would be provided by a segmented deformable mirror matched to the fiber array, dispersion control by fiber-stretching units, and amplitude control by adjusting the power coupled into each fiber by slight input pointing/coupling changes. A potential mission architecture resulting from these considerations is shown in Fig. 6, including also the needed wavefront sensing step, and the active fiber control step, for which piezoelectric fiber length matching (stretching) units are envisioned (e.g., Fig. 7).

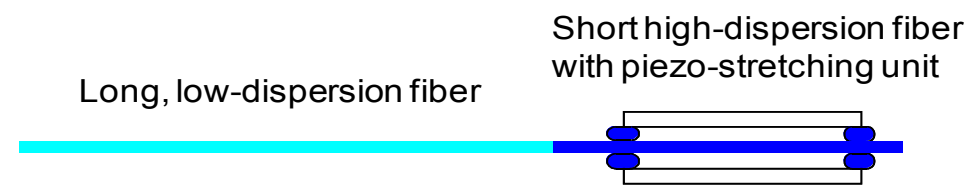

Figure 7. Potential fiber compensation architecture.

The active control over each element of the pupil in both amplitude and phase essentially results in an optical phased array, analogous to the case of a radio phased array. Moreover, the intensity control over each element in the array would allow apodization of the light emerging from the fiber array (Fig. 6), similar to the case of classical apodization, but with discrete sampling, thus in principle enabling a high-contrast image without any further stellar suppression steps. Due to the similarity of this controlled aperture approach to radio phased arrays, this visible-wavelength approach is referred to as the "phased array coronagraph" (PAC) ${ }^{21}$.

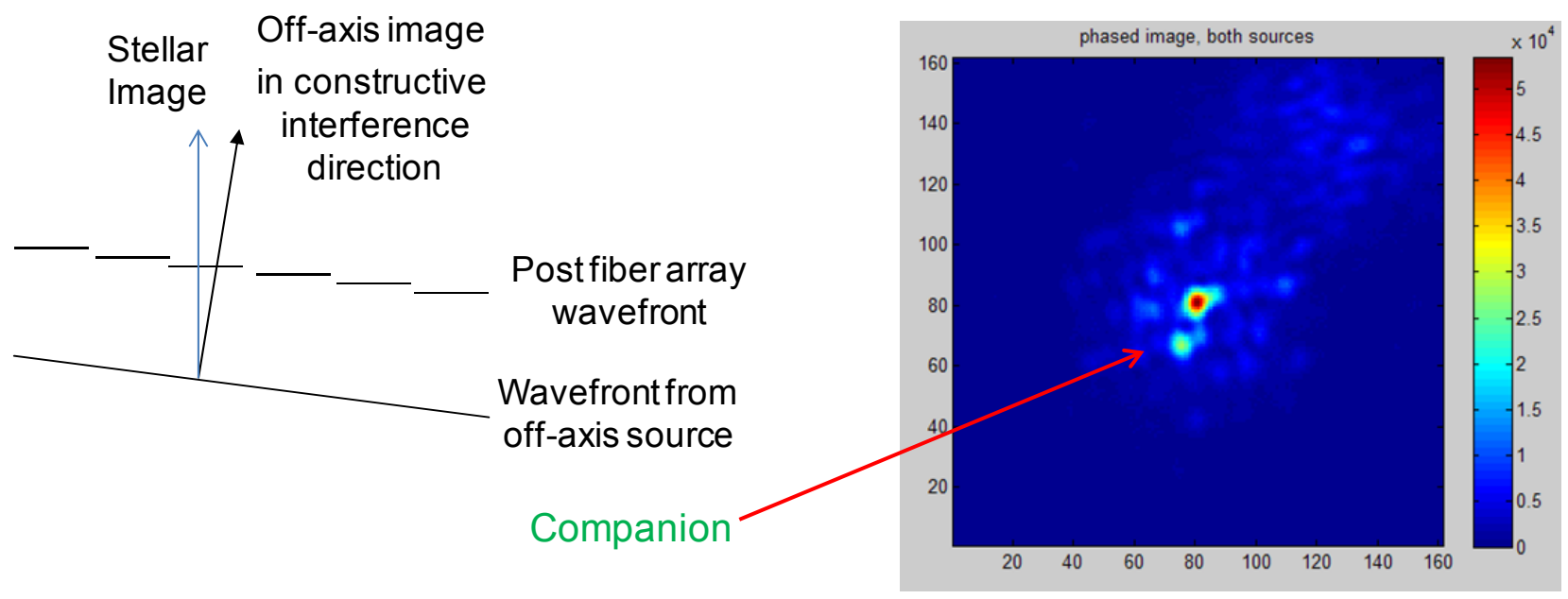

Figure 8. Left: Image formation for an on-axis star and a companion through an array of fibers. Right: Demonstration of companion detection through an array of single mode fibers. The companion is within an individual fiber's single mode field of view (the light blue speckled region covering roughly the central $1 / 3$ of the image).

Fig. 6 also shows both the calculated pupil-plane distribution and the focal-plane image for the case of an ideal PAC. The plot at the bottom of Fig. 6 gives the calculated contrast along the image diagonal, demonstrating that an ideal PAC can, in theory, reach the desired $10^{-10}$ off-axis contrast level.

The requirements imposed upon a fiber array to reach this level of performance are of course rather demanding. The monochromatic phasing requirement is the same as for any other high-contrast coronagraph, of order several tens of pm for a large array, a phasing requirement that would need to be met by the system's deformable mirror. Dispersion 
matching sets a condition on fiber length matching of $\sigma / \Delta \mathrm{n}$, where $\sigma$ is the final rms wavefront error required and $\Delta \mathrm{n}$ is the change in the fiber's refractive index across the band, of order $\sim 0.1$ to $1 \mu \mathrm{m}$ for various classes of fiber. Low dispersion fibers are thus a must, and moreover the fibers would likely also need to be polarization-maintaining or selective, with low temperature sensitivity. New types of photonic crystal fiber may be most appropriate, especially lowdispersion hollow core fibers. A detailed error analysis is presented by Martin et al. in these proceedings.

Laboratory demonstrations of this fiber-array-based imaging approach have been initiated on an experimental testbed in our lab at JPL, and as Fig. 8 shows, an off-axis companion to a bright on-axis source can indeed be seen in an image acquired through our single-mode fiber array. As both of the imaged sources seen in Fig. 8 were within the individual fibers' single-mode field of view, this result demonstrates that companions to a bright central source can indeed be retrieved inside the single-mode field of view of an individual fiber.

\section{SUMMARY}

Over the past decade or so, interferometric and imaging uses of fibers in astronomy have shown significant advances. In interferometry, single-mode fibers have enabled high-accuracy visibility and null depth measurements, and integrated optics beam combiners are also beginning to play a role. The role of multi-fiber arrays is not yet as advanced, but several new concepts have emerged, and fibers are beginning to play a role in on-sky pupil masking observations. In the laboratory, recovery of an off-axis source from pupil-masking visibility measurements has been demonstrated, large single-mode fiber arrays are being developed, and one such fiber array has recently been used to demonstrate direct imaging within an individual fiber's single mode field of view for the first time.

This work was carried out at the Jet Propulsion Laboratory, California Institute of Technology, under contract with the National Aeronautics and Space Administration.

\section{REFERENCES}

[1] Marois C, et al., Nature 468, 1080 (2010)

[2] Meinel, M.B. \& Meinel, M.P., Applied Optics 43, 601 (2004)

[3] Mennesson, B., Ollivier, M. and Ruilier, C., J. Opt. Soc. Am. A 19, 596 (2002)

[4] Vasisht, G., Booth, A.J., Colavita, M.M., Johnson, R.L., Ligon, E.R., Moore, J.D. and Palmer, D.L., Proc. SPIE 4838, 824 (2003)

[5] Coude du Foresto, V., Perrin, G., Ruilier, C., Mennesson, B., Traub, W. and Lacasse, M., Proc. SPIE 3350, 856 (1998)

[6] Berger, J.P. et al., Proc. SPIE 7734, 773435-1 (2010)

[7] Wallner O., Armengol, J. M. P. and Karlson, A.L., Proc. SPIE 5491, 798 (2004)

[8] Haguenauer, P. and Serabyn, E., Applied Optics, 45, 2749 (2006)

[9] Martin, S., Serabyn, E., Liewer, K., Loya, F., Mennesson, B., Hanot, Ch. \& Mawet, D., Proc. SPIE 7013, 70131Y (2008)

[10] Hanot, C., Mennesson, B., Martin, S., Liewer, K., Loya, F., Mawet, D., Riaud, P., Absil, O. \& Serabyn, E., Astrophys. J. 729, 110 (2011)

[11] Bracewell, R. N., Nature 274, 780 (1978)

[12] Serabyn, et al., Proc. SPIE 7734, 77341E (2010)

[13] Kraus, A. L., Ireland, M. J., Martinache, F. and Lloyd, J.P., Astrophys. J. 679, 762 (2008)

[14] Perrin, G., Lacour, S. Woillez, J. and Thiebaut, E., Mon. Not. Roy. Ast. Soc. 373, 747 (2006)

[15] Kotani, T. et al., Proc. SPIE 7014, 70141P-1 (2008)

[16] Kotani T., Lacour, S. Perrin, G., Robertson, G. and Tuthill, P., Opt. Exp. 17, 1925 (2009)

[17] Mennesson, B., Shao, M., Levine, B.M., Wallace, J.K., Liu, D.T., Serabyn, E., Unwin, S.C. and Beichman, C.A., Proc. SPIE 4860, 32 (2003)

[18] Liu, D., Levine, B.M., Shao, M. and Aguayo, F., Proc. 2005 IEEE Aerospace Conf., DOI 10.1109 (2005)

[19] Sandhu, J., Irwin, P., Shao, M., Shaw, J., Smythe, R., Levine, B., Lyon' R., BAAS Vol 43, No. 2, 253.11 (2011)

[20] Lyon, R.G. et al., Proc. SPIE 7731, 77312B-1 (2010)

[21] Serabyn, E., Astrophys. J. 697, 1334 (2009)

Proc. of SPIE Vol. $8146 \quad 81460 \mathrm{l}-7$ 